\title{
Mapping the evidence on health equity considerations in economic evaluations of health interventions: a scoping review protocol
}

\author{
Hafizah Besar Sa'aid ${ }^{1,2,3^{*}}$ (D), Sharon Mathew ${ }^{3}$, Marina Richardson ${ }^{1,3}$, Joanna M. Bielecki ${ }^{1}$ and Beate Sander ${ }^{1,3,4,5}$
}

\begin{abstract}
Background: Equity in health has become an important policy agenda around the world, prompting health economists to advance methods to enable the inclusion of equity in economic evaluations. Among the methods that have been proposed to explicitly include equity are the weighting analysis, equity impact analysis, and equity trade-off analysis. This is a new development and a comprehensive overview of trends and concepts of health equity in economic evaluations is lacking. Thus, our objective is to map the current state of the literature with respect to how health equity is considered in economic evaluations of health interventions reported in the academic and gray literature.
\end{abstract}

Methods: We will conduct a scoping review to identify and map evidence on how health equity is considered in economic evaluations of health interventions. We will search relevant electronic, gray literature and key journals. We developed a search strategy using text words and Medical Subject Headings terms related to health equity and economic evaluations of health interventions. Articles retrieved will be uploaded to reference manager software for screening and data extraction. Two reviewers will independently screen the articles based on their titles and abstracts for inclusion, and then will independently screen a full text to ascertain final inclusion. A simple numerical count will be used to quantify the data and a content analysis will be conducted to present the narrative; that is, a thematic summary of the data collected.

Discussion: The results of this scoping review will provide a comprehensive overview of the current evidence on how health equity is considered in economic evaluations of health interventions and its research gaps. It will also provide key information to decision-makers and policy-makers to understand ways to include health equity into the prioritization of health interventions when aiming for a more equitable distribution of health resources.

Systematic review registration: This protocol was registered with Open Science Framework (OSF) Registry on August 14, 2019 (https://osf.io/9my2z/registrations).

Keywords: Health equity, Economic evaluations, Health interventions, Scoping review, Decision-making

\footnotetext{
* Correspondence: Hafizah.Saaid@uhnresearch.ca; fizah598@uitm.edu.my

${ }^{1}$ Toronto Health Economics and Technology Assessment (THETA)

Collaborative, University Health Network, Toronto, Ontario, Canada

${ }^{2}$ Faculty of Business and Management, Universiti Teknologi MARA (UiTM),

Sungai Petani, Kedah, Malaysia

Full list of author information is available at the end of the article
}

(C) The Author(s). 2020 Open Access This article is distributed under the terms of the Creative Commons Attribution 4.0 International License (http://creativecommons.org/licenses/by/4.0/), which permits unrestricted use, distribution, and reproduction in any medium, provided you give appropriate credit to the original author(s) and the source, provide a link to the Creative Commons license, and indicate if changes were made. The Creative Commons Public Domain Dedication waiver (http://creativecommons.org/publicdomain/zero/1.0/) applies to the data made available in this article, unless otherwise stated. 


\section{Background}

Maximization of health, reduction of inequities in health, and financial protection against the costs of ill health are three central tenets of priority setting for health interventions [1]. However, the present priority setting methods often focus on health maximization and do not adequately capture the complexities of equity and financial protection [1]. The goal of achieving equity in health has become central to the post-2015 United Nations' (UN) Development Agenda [2] and the UN's 2030 Agenda for Sustainable Development [3]. The influence of these mandates is evident in advances in the methods of economic evaluation of health interventions, where health equity has been introduced as an important consideration [4]. However, a comprehensive study that maps the trends and concepts of health equity in health economic evaluations of health interventions has yet to be conducted. In response to this perceived gap, our objective is to map the existing knowledge on how health equity is considered in economic evaluations of health interventions as reported in the academic and gray literature.

The objective of this protocol is to provide the rationale, scope, and methods for the conduct of the scoping review. This protocol will start with the background information: the definition of health equity, why equity is important to be considered in prioritizing the health interventions, and how equity is an important consideration in health economic evaluations.

\section{Health equity: definition and importance}

Equity is grounded in principles of human rights and distributive justice [5]. The concept of equity is intrinsically normative (value-based), and "... a matter of morality, fairness and justice" [6]. Consequently, health equity refers to the absence of unjust or unfair health disparities, i.e., preventable or remediable differences in one or more determinants of health across populations or population groups [7, 8]. The WHO elaborates further that "Health inequities involve more than inequality with respect to health determinants, access to resources needed to improve and maintain health and health outcomes", and thus health equity refers to the fair distribution of health in the population.

Health equity can be divided into horizontal and vertical equity. Horizontal equity means equal access to health for all population groups (i.e., people of equal needs should be treated the same), whereas vertical equity means inclusion of all needed services (i.e., people with greater clinical needs should have more intervention than those with fewer needs) $[9,10]$. Equity is an important topic on the world policy agenda because of disparities in health among population groups (between rich and poor, urban and rural, employed and unemployed) and countries (between low-, middle- and high-income countries).
Equity has been at the forefront of the WHO's agenda since 1946. In 1978, participant countries at the AlmaAta WHO Conference endorsed the concept of health equity, which resulted in launching the "Health for All (HFA)" campaign [11]. Since then, equity in health has become an important part of the policy agenda for all countries. In 2000, eight Millennium Development Goals (MDGs) were established by the United Nations (UN) to address health inequity by 2015 [3]. As continuity, the UN launched the 17 Sustainable Development Goals (SDGs) that need to be achieved by 2030. In these goals, health equity has become the soul of the agenda [3].

Despite the declared commitment to health equity, most health systems around the world (from macro to micro) are still routinely making decisions on choosing new health interventions based on economic evaluations and maximizing health outcomes, often measured in qualityadjusted life-years (QALYs) [12, 13]. Hence, decisionmakers intrinsically balance quality of care and financial outcome parameters [14], but rarely make decisions based on the trade-offs between the economic and equity distributions of the health outcomes [4].

\section{Health equity as a part of health economic evaluations}

Nevertheless, the rise of health equity on the policy agenda around the world has triggered health economists to develop methods to explicitly include equity in health economic evaluations of new health interventions in order to help policy- and decision-makers make informed decisions with an equity focus. Among the methods that have been proposed to quantify health inequity are rate ratios, population attributable risks, slope and relative indices of inequality, and the concentration curve and index [15]. Other methods include the equity weighting analysis [16], equity impact analysis, and equity trade-off analysis [4]. Still, until today, decision-making bodies have yet to adopt these methods to consider equity in their decisions. If decisionmakers consider equity, it is usually informal, ad hoc, simplistic and lacking transparency [16]. Evidence on how to improve health equity in the most efficient way is vital [17].

Since the 1978 Declaration of Alma-Ata, citations in MEDLINE that include the term "equity" increased by $216 \%$ in 2015 compared to 1980 [18]. Given the increase in publications, comprehensive reviews and analyses on equity consideration in economic evaluations have also been conducted, though most reviews have focused on specific methodologies of how to integrate equity concerns into economic evaluations [19-21]. Other reviews have been based on specific interventions or concepts, such as vaccines, operation research, and universal health coverage [9, 18, 22]. However, no review has comprehensively mapped the existing knowledge to date in a scoping review.

Scoping reviews follow a rigorous and transparent process in identifying relevant literature to map the 
broad knowledge instead of summing up the best available research on a specific question [23]. In our scoping review, we will map the current state of trends and concepts of how health equity is considered in health economic evaluations both empirically and methodologically, and synthesize the literature to identify any knowledge gaps.

\section{Methods}

\section{Study design}

Our protocol is guided by the scoping review framework proposed by Arksey and O'Malley, which was refined further by Levac et al. and the Joanna Briggs Institute [24-26]. The overview of the stages for the scoping review process as proposed by Arksey and O'Malley is depicted in Table $1[24,25]$. The populated PRISMA-P checklist [27] for this study protocol is attached as an additional file (see Additional file 1).

The original framework proposed by Arksey and O'Malley [24] comprised six stages, including an optional consultation with key stakeholders to identify additional references and gather feedback on the findings of the scoping review. While a consultation with stakeholders would be a valuable exercise, we will not include that optional stage in this scoping review because of budget and time constraints. Our protocol was registered with Open Science Framework (OSF) Registry on August 14, 2019 (https://osf.io/9my2z/registrations).

\section{Stage 1: identifying the research question}

The purpose of this scoping review is to map the current state of the literature on the trends and concepts of how health equity is considered in health economic evaluations of health interventions, and synthesize the literature to identify knowledge gaps. We will include methods papers and empirical studies that consider health equity in full economic evaluations such as cost-utility analysis, cost-effectiveness analysis, cost-benefit analysis, and cost-minimization analysis.

To identify our research questions, we conducted an exploratory review of the literature on health equity in health economic evaluations of health interventions. Based on this exploratory review of the literature, our overarching research question is "What is known in the literature about how health equity is considered in economic evaluations of health interventions?" and our specific research questions are:

1. How is health equity incorporated in economic evaluations of health interventions?

2. For which types of health interventions is health equity included as part of the economic evaluations?

3. How is health equity conceptualized in the economic evaluations of health interventions?

4. Which types of economic evaluations consider health equity in the assessment of health interventions?

5. Which methods are currently used to incorporate health equity in economic evaluations of health interventions?

6. In which settings is health equity considered in economic evaluations of health interventions?

7. Which new methods are proposed for incorporating health equity in economic evaluations of health interventions?

8. Did the conceptualization of health equity considered in economic evaluations of health interventions change over time? If so, how?

9. What are the knowledge gaps in incorporating health equity in economic evaluations of health interventions?

Table 1 The original scoping review framework proposed by Arksey and O'Malley [24, 25]

\begin{tabular}{|c|c|c|}
\hline \multicolumn{2}{|c|}{ Stage } & \multirow{2}{*}{$\begin{array}{l}\text { Description } \\
\text { - As a guide to build a research strategy. } \\
\text { - Should be wide enough to generate a breadth of coverage. }\end{array}$} \\
\hline 1. & Identifying the research question & \\
\hline 2. & Identifying relevant studies & $\begin{array}{l}\text {-Via different sources: electronic databases, reference lists, hand-searching } \\
\text { of key journals, existing networks, relevant organizations, and conferences. } \\
\text { - Make decision about the coverage of the review in terms of time span } \\
\text { and language (consider time and budget constraints). }\end{array}$ \\
\hline 3. & Study selection & $\begin{array}{l}\text { - The criteria for the inclusion/exclusion criteria are device post hoc based } \\
\text { on increasing familiarity with the subject matter through reading the studies. } \\
\text { - Use an iterative team approach to select the studies and extract the data. }\end{array}$ \\
\hline 4. & Charting the data & $\begin{array}{l}\text { - The work involves "charting" key items of the information obtained from } \\
\text { the primary research reports being reviewed. } \\
\text { - A data-charting form is developed and used to extract data from each study. }\end{array}$ \\
\hline 5. & Collating, summarizing, and reporting the results & $\begin{array}{l}\text { - An analytic framework or thematic construction is used to provide an } \\
\text { overview of the breadth of the literature but not a synthesis. } \\
\text { - A numerical analysis of the extent and nature of the studies using tables } \\
\text { and charts is presented. A thematic analysis is then presented. } \\
\text { - Clarity and consistency are required when reporting results. }\end{array}$ \\
\hline 6. & Consultation exercise (optional) & $\begin{array}{l}\text { - Provides opportunities for consumer and stakeholder involvement to suggest } \\
\text { additional references and provide insights beyond those in the literature. }\end{array}$ \\
\hline
\end{tabular}




\section{Stage 2: identifying relevant studies}

Based on the Joanna Briggs Institute Reviewers' Manual 2015 (Methodology for JBI Scoping Reviews) [26], the aim for the second stage of scoping reviews is to identify the criteria that will be used to select the studies, i.e., inclusion criteria to guide the search and help filter for relevant sources.

We searched the following eight databases for relevant articles: MEDLINE Ovid, EMBASE Ovid, EconLit EBSCO, Scopus Elsevier, CINAHL EBSCO, Cochrane Database of Systematic Reviews (CDSR), Proquest (Thesis and Dissertations), and Proquest University of Toronto (Thesis and Dissertations) from the date of inception to May 2019. We have not limited our analysis to economic evaluations published in journals or publications that have undergone formal review as study quality is not assessed for scoping reviews $[24,26]$. We also searched for gray literature by following the methods outlined in "Grey Matters: a practical tool for searching health-related grey literature" [28].

The search strategy was designed by a medical research librarian (JB) and it consists of both text words and Medical Subject Headings (MeSH) terms related to economic evaluations and equity. We conducted the search in English. We used published and validated filters to search the following conceptual areas:

1. Health equity (equity, inequity, social determinants of health, health disparity) [29].

2. Economic evaluation (decision model, cost-effectiveness, cost-utility, cost-benefit, pharmaco-economics, cost minimization, cost-efficiency analysis) [30].

Further, we will screen the articles retrieved based on their titles and abstracts, and then a full-text. For full-text screening, we will make every effort to screen all included full-text papers in any language. We will search the reference lists of included full-text studies and hand search key journals to identify any other relevant publications. The articles retrieved will be uploaded to DistillerSR, a reference manager software, which will be also used for data management for further screening and data extraction.

Based on the initial exploratory search and discussions among the review team members and the medical research librarian (JB), our inclusion criteria are:

- Source of information: Publications that are accessible publicly and through University of Toronto and University Health Network libraries

- Time frame: From inception to May 2019

- Language: Abstracts in English language; full-text in any languages

- Research location: Worldwide

- Study population: Human
- Types of interventions: All types of health interventions (medical devices, drugs, procedures, treatments, public health interventions, etc.)

- Type of economic evaluations: Full economic evaluations (cost-effectiveness analysis, cost-utility analysis, cost-minimization analysis, and cost-benefit analysis)

- Type of methods incorporating equity: Any methods of incorporating health equity in economic evaluations (rate ratios, equity weighting analysis, equity impact analysis, equity trade-off analysis, etc.)

- Type of studies: Both empirical (applied) and methods (concept) studies

- Types of articles: All types of study designs, applied studies, concept discussion papers, reviews, meta-analyses, books, theses and dissertations, gray literature, and general articles (including commentaries or editorial articles)

The search strategy for MEDLINE Ovid, together with CLHTA, CLEED, and CDSR, and gray literature are included as additional files (see Additional file 2 and Additional file 3).

\section{Stage 3: study selection}

Selection will be performed based on the inclusion criteria pre-specified in stage 2 of the review protocol and will be conducted in the following two-step process.

Step 1: We will import and de-duplicate the search results from all sources in DistillerSR. Two reviewers will conduct title and abstract screening. All types of articles on health equity considerations in economic evaluations of health interventions as per inclusion criteria in stage 2 will be included. The reviewers will screen each article based on the following question: "Does the article discuss on how health equity is considered in economic evaluations of health intervention?"

To increase consistency among reviewers, all reviewers will first screen the same 100 titles and abstracts and discuss conflicts until a consensus is reached. If consensus cannot be reached, conflicts will be brought to the larger team for further discussion. The reviewers will then screen the remaining citations independently (two reviewers per article) and remove irrelevant studies. For those fulfilling the eligibility criteria, the full-text articles will be retrieved.

Step 2: The full-text articles will be screened independently by the reviewers (two reviewers per article) against the inclusion/exclusion criteria and the rationale for exclusion will be documented. Any eligibility discrepancies will be discussed between reviewers until consensus is reached or will be brought to the larger team for discussion if consensus cannot be reached. We will exclude book reviews, letters, and conference abstracts. Studies remaining at the end of Stage 2 will be included 
in the review and a rationale for inclusion will be provided.

\section{Stage 4: charting the data}

In stage 4, we will develop a standardized data extraction template to allow the reviewers to categorize or "chart" the data. There are six main categories and 26 subcategories that will be used to assess the full review of the articles that are retrieved after fulfilling the eligible inclusion criteria. We will extract the standard bibliographical information (such as authors, title, journal, and year of publication), study details (such as study designs, data type, settings, and outcomes-_-if applicable), and what, why, and how health equity was considered (Table 2).

The reviewers will pilot test the template on a sample of the included studies (i.e., 10\% of the included studies) to make sure that the charting is consistently applied. If

Table 2 Data extraction charting template

\begin{tabular}{|c|c|}
\hline Main category/subcategories & Description \\
\hline \multicolumn{2}{|l|}{ Article details } \\
\hline Author(s) & Who are the author(s)? \\
\hline Title & Full title of the article \\
\hline Objective(s) & Describe the stated objective(s) \\
\hline Year of publication & Article year \\
\hline Article type & What is the type of article? \\
\hline Language & In which language is the article written? \\
\hline Country & In which country was the study conducted? \\
\hline \multicolumn{2}{|l|}{ Study details (if applicable) } \\
\hline Type of study & Empirical or methods? \\
\hline Study design & Type of methodology \\
\hline Data type & Primary or secondary data \\
\hline Study population & Which population was studied? \\
\hline \multicolumn{2}{|l|}{ Health intervention } \\
\hline Name & Name of the intervention(s) \\
\hline Type of intervention & $\begin{array}{l}\text { What was the type of intervention? (e.g., vaccine, drugs, medical devices, treatment, procedure, and public } \\
\text { health) }\end{array}$ \\
\hline Settings & Where did the intervention/study take place? \\
\hline \multicolumn{2}{|l|}{ Health equity } \\
\hline Reasons & Why did the study assess health equity? \\
\hline Type of health equity & $\begin{array}{l}\text { Which type of health equity was considered? (e.g., horizontal and vertical equity, equal utilization, distribution } \\
\text { according to need, equal access, and equal health outcomes) }\end{array}$ \\
\hline $\begin{array}{l}\text { Social determinants of health (if } \\
\text { applicable) }\end{array}$ & Which social determinants of health were addressed? \\
\hline Other equity measures & Which other equity measures were addressed? (e.g., demographic factors and financial risk protection (FRP)) \\
\hline Evaluation method & $\begin{array}{l}\text { How was health equity assessed? (e.g., rate ratios, population attributable risks, equity weighting analysis, equity } \\
\text { impact analysis, and equity trade-off analysis) }\end{array}$ \\
\hline Tool used & Which tool was used or developed to assess health equity? (e.g., framework and guidelines) \\
\hline \multicolumn{2}{|l|}{ Health economic evaluations } \\
\hline Type of evaluations & Which type of economic evaluation was conducted? \\
\hline Evaluation method & How was the economic evaluation conducted? (e.g., administrative data, and clinical trials) \\
\hline Tool used & Which tool was used or developed? (e.g., framework and guidelines) \\
\hline \multicolumn{2}{|l|}{ Incorporating health equity } \\
\hline Method development & How does health equity incorporated in the economic evaluations of health interventions? \\
\hline New method & What are the new methods proposed? \\
\hline Method practicality & Are there any empirical studies that used the method proposed? \\
\hline Recommendations & What recommendations did the authors make based on the study results and their experiences? \\
\hline
\end{tabular}


necessary, we will refine and revise the template accordingly through the course of the review process. If questions arise during the pilot test, the team will resolve the issues through discussion. The same reviewers will extract and chart the data from each included article independently (two reviewers per article) based on the data extraction template. We will make every effort to identify native speakers which can extract the data of all included studies published in languages other than English.

Stage 5: collating, summarizing, and reporting the results The data analysis will provide an overview of the breadth of research which in turn will inform of the current status and the way forward in considering health equity in economic evaluations of health interventions. We will follow the PRISMA-ScR checklist [31] and Arksey and O'Malley's [24] methods of reporting to provide a descriptive numerical analysis of the topic, including the extent, characteristics, and distribution of the included studies. The PRISMA flow diagram for the scoping review process, as suggested by the Joanna Briggs Institute [26] in their scoping review manual, will also be observed. We will first provide a narrative; that is, a thematic summary of the data collected. We will then discuss the results based on the research questions and purpose of the study. We will also highlight areas that are underresearched and may need further investigation.

We will report trends over time for methods and empirical studies of health equity considerations in economic evaluations of health interventions. We will explore gaps between the conceptual thinking about health equity and the application of these methods. We will present the results in aggregate and visual forms (e.g., tables, charts, evidence and concept maps, and bubble plots) as appropriate.

\section{Discussion and dissemination}

We anticipate that the results of this scoping review will provide a comprehensive overview of the evidence on health equity considerations in economic evaluations of health interventions and identify research gaps. The review will also provide key information to decisionmakers and policy-makers at the macro and micro levels to understand ways of including health equity into the prioritization of health interventions when aiming for a more equitable distribution of health resources. We will disseminate the findings of this scoping review through publication in a scientific journal and presentation at relevant conferences. This protocol reports a comprehensive and transparent methodology that will inform a rigorous review of equity considerations in economic evaluations of health interventions and lay a foundation for future work in the field.

\section{Supplementary information}

Supplementary information accompanies this paper at https://doi.org/10. 1186/s13643-019-1257-4.

Additional file 1. PRISMA-P Checklist. (docx 37kb)

Additional file 2. Search strategy for Ovid MEDLINE, CLHTA + CLEED + CDSR. (docx 21kb)

Additional file 3. Search strategy for grey literature. (docx 16kb)

\section{Abbreviations}

HFA: Health for All; MDGs: Millennium Development Goals; MeSH: Medical Subject Headings; PRISMA: Preferred Reporting Items for Systematic Reviews and Meta-Analyses; PRISMA-P: Preferred Reporting Items for Systematic Reviews and Meta-Analyses - Protocol; PRISMA-ScR: Preferred Reporting Items for Systematic Reviews and Meta-Analyses - Scoping Review; QALYs: Quality-adjusted life-years; SDGs: Sustainable Development Goals; UN: United Nations; WHO: World Health Organization

\section{Acknowledgements}

Not applicable

\section{Authors' contributions}

HBS conceived the research. HBS and SB designed the research questions. $H B S, S B$, and JB developed and refined the search strategy. HBS, MR, and SM performed the initial screening of the articles. HBS prepared and drafted the manuscript. All the authors edited and revised the manuscript. All the authors read and approved of the final manuscript.

\section{Funding}

Hafizah Besar Sa'aid is funded by the Ministry of Education Malaysia Postdoctoral Fellowship at the Toronto Health Economics and Technology Assessment (THETA) Collaborative at the University of Toronto.

\section{Availability of data and materials}

Data sharing is not applicable to this article as no datasets were generated or analyzed during the current study.

\section{Ethics approval and consent to participate}

Ethics approval and consent to participate is not applicable to this article as no involvement from human or animal population during the current study.

\section{Consent for publication}

Not applicable for the current study because there is no individual person's data in this manuscript.

\section{Competing interests}

The authors declare that they have no competing interests.

\section{Author details}

${ }^{1}$ Toronto Health Economics and Technology Assessment (THETA) Collaborative, University Health Network, Toronto, Ontario, Canada. ${ }^{2}$ Faculty of Business and Management, Universiti Teknologi MARA (UiTM), Sungai Petani, Kedah, Malaysia. ${ }^{3}$ Institute of Health Policy, Management and Evaluation, University of Toronto, Toronto, Ontario, Canada. ${ }^{4}$ Public Health Ontario, Toronto, Ontario, Canada. ${ }^{5}$ ICES, Toronto, Ontario, Canada.

Received: 23 August 2019 Accepted: 18 December 2019

Published online: 08 January 2020

\section{References}

1. Norheim OF, Baltussen R, Johri M, Chisholm D, Nord E, Brock DW, et al. Guidance on priority setting in health care (GPS-health): the inclusion of equity criteria not captured by cost-effectiveness analysis. BioMed Central: Cost Eff Resour Alloc 2014; doi:https://doi.org/10.1186/1478-7547-12-18.

2. United Nation. Department of Economic and Social Affairs. Millennium Development Goals Report. 2012; https://www.un.org/en/development/ desa/publications/mdg-report-2012.html. 
3. UN General Assembly, Transforming our world: the 2030 Agenda for Sustainable Development, 21 October 2015. https:/www.refworld.org/ docid/57b6e3e44.html

4. Cookson R, Mirelman AJ, Griffin S, Asaria M, Dawkins B, Norheim OF, et al. Using cost-effectiveness analysis to address health equity concerns. Value Health. 2017;20:206-12.

5. Braveman P, Gruskin S. Defining equity in health. J Epidemiol Community Health. 2003. https://doi.org/10.1136/jech.57.4.254.

6. Fee E, Gonzalez AR. The history of health equity: concept and vision. Diversity and Equality in Health and Care. 2017;14(3):148-52.

7. World Health Organization (WHO). Health Systems: Equity. 2019. https:// www.who.int/healthsystems/topics/equity/en/. Accessed 26 April 2019.

8. Stewart AL, Napoles-Springer AM. Advancing health disparities research: can we afford to ignore measurement issues? Med Care. 2003;41(11):1207-20.

9. Rodney AM, Hill PS. Achieving equity within universal health coverage: a narrative review of progress and resources for measuring success. Rodney and Hill Int J Equity Health 2014;13:72.

10. Raine R, Fitzpatrick R, Barratt H, Bevan G, Black N, Boaden R, et al. Challenges, solutions and future directions in the evaluation of service innovations in health care and public health. Health Serv Deliv Res. 2016;4(16):69-84.

11. World Health Organization (WHO). Global strategy for Health for All by the year 2000. 1981. https://iris.wpro.who.int/bitstream/handle/10665.1/6967/ WPR_RC032_GlobalStrategy_1981_en.pdf.

12. Thokala P, Duenas A. Multiple criteria decision analysis for health technology assessment. Value Health. 2012;15(8):1172-81.

13. Hauck K, Thomas R, Smith PC. Departures from cost-effectiveness recommendations: the impact of health system constraints on priority setting. Health Syst Reform. 2016;2(1):61-70.

14. Gurtner S. Making the right decisions about new technologies: a perspective on criteria and preferences in hospital. Health Care Manag Rev. 2014;39(3):245-54.

15. Braveman P. Health disparities and health equity: concepts and measurement. Annu Rev Public Health. 2006;27:167-94.

16. Round J, Paulden M. Incorporating equity in economic evaluations: a multiattribute equity state approach. Eur J Health Econ. 2018;19:489-98.

17. Welch VA, Petticrew M, O'Neill J, Waters E, Armstrong R, Bhutta ZA, et al. Health equity: evidence synthesis and knowledge translation methods. Syst Rev. 2013;2:43

18. Boujaoude M-A, Mirelman AJ, Dalziel K, Carvalho N. Accounting for equity considerations in cost-effectiveness analysis: a systematic review of rotavirus vaccine in low and middle-income countries. Cost Eff Resour Alloc. 2018;16:18.

19. Lal A, Moodie M, Peeters A, Carter R. Inclusion of equity in economic analyses of public health policies: systematic review and future directions. Aust N Z J Public Health. 2018;42(2):207-13.

20. Dukhanin V, Searle A, Zwerling A, Dowdy DW, Taylora HA, Merritte MW. Integrating social justice concerns into economic evaluation for health and public health: a systematic review. Soc Sci Med. 2018;198:27-35.

21. Johri M, Norheim OF. Can cost-effectiveness analysis integrate concerns for equity? Systematic review. Int J Technol Assess Health Care. 2012;28(2):125-32.

22. Bradley BD, Jung T, Tandon-Verma A, Khoury B, Chan TCY, Cheng Y-L. Operations research in global health: a scoping review with a focus on the themes of health equity and impact. Health Res Policy Syst. 2017;15:32.

23. Shen N, Sockalingam S, Abi Jaoude A, Bailey SM, Bernier T, Freeland A, et al. Scoping review protocol: education initiatives for medical psychiatry collaborative care. BMJ Open. 2017;7(9):e015886.

24. Arksey H, O'Malley L. Scoping studies: towards a methodological framework. Int J Soc Res Methodol: Theory Pract. 2005;8(1):19-32.

25. Levac D, Colquhoun H, O'Brien KK. Scoping studies: advancing the methodology. Implement Sci. 2010;5:69.

26. The Joanna Briggs Institute. Joanna Briggs Institute Reviewers' Manual: 2015 edition/supplement. Adelaide: The Joanna Briggs Institute; 2015. https:// nursing.lsuhsc.edu/JBI/docs/ReviewersManuals/Scoping-.pdf.

27. Shamseer L, Moher D, Clarke M, Ghersi D, Liberati A, Petticrew M, et al. The PRISMA-P Group. Preferred Reporting Items for Systematic Review and Meta-Analysis Protocols (PRISMA-P) 2015: elaboration and explanation. BMJ. 2015;349:g7647.

28. Canadian Agency for Drugs and Technologies in Health (CADTH). Grey Matters: a practical tool for searching health-related grey literature (Internet). Ottawa: 2018. https:/www.cadth.ca/resources/finding-evidence/grey-matters. Accessed 23 May 2019.
29. Prady SL, Uphoff EP, Power M, Golder S. Development and validation of a search filter to identify equity-focused studies: reducing the number needed to screen. BMC Med Res Methodol. 2018;18:106.

30. Glanville J, Fleetwood K, Yellowlees A, Kaunelis D, Mensinkai S. Development and testing of search filters to identify economic evaluations in MEDLINE and EMBASE. Ottawa: Canadian Agency for Drugs and Technologies in Health; 2009. https://www.cadth.ca/media/pdf/H0490_ Search_Filters_for_Economic_Evaluations_mg_e.pdf.

31. Tricco AC, Lillie E, Zarin W, O'Brien KK, Colquhoun H, Levac D, et al. PRISMA extension for scoping reviews (PRISMA-SCR): checklist and explanation. Ann Intern Med. 2018;169(7):467-73.

\section{Publisher's Note}

Springer Nature remains neutral with regard to jurisdictional claims in published maps and institutional affiliations.
Ready to submit your research? Choose BMC and benefit from:

- fast, convenient online submission

- thorough peer review by experienced researchers in your field

- rapid publication on acceptance

- support for research data, including large and complex data types

- gold Open Access which fosters wider collaboration and increased citations

- maximum visibility for your research: over $100 \mathrm{M}$ website views per year

At BMC, research is always in progress.

Learn more biomedcentral.com/submissions 